\title{
CONTRIBUIÇÃO DA PANDEMIA DE COVID-ı9 PARA O AUMENTO DOS CASOS DE SÍNDROME DE BURNOUT EM PROFISSIONAIS DE ENFERMAGEM
}

\author{
Natália Duarte Marcos ${ }^{1}$ \\ Tiago Pacheco Brandão Ribeiro ${ }^{2}$
}

RESUMO: O presente estudo surge a partir da necessidade de investigar a saúde mental dos profissionais de enfermagem diante o enfrentamento da COVID-I9, objetivando caracterizar o esgotamento profissional e identificar as características envolvidas no trabalho da enfermagem na linha de frente do combate à pandemia que possam influenciar na ocorrência da Síndrome de Burnout. A presente pesquisa constata que sintomas de depressão, ansiedade e estresse têm sido frequentes entre esses trabalhadores, logo, promover a saúde dos profissionais é um assunto de extrema relevância, uma vez que estes indivíduos precisam estar bem para serem capazes de prestar um serviço de qualidade à população. É necessário implementar medidas de intervenção psicológica, tais como: reorganização da jornada de trabalho (permitindo tempo para descanso); valorização e reconhecimento pelo serviço prestado; melhora na remuneração; fornecimento adequado de EPIs e auxílio psicológico para promover o bem-estar mental e uma melhor qualidade de vida aos profissionais de saúde expostos durante a pandemia. Conclui-se que é de suma importância explorar as possíveis formas de se evitar o Burnout, uma vez que o enfermeiro é um profissional de fundamental importância para o combate ao novo coronavírus.

Palavras-chave: Enfermagem. Síndrome de Burnout. Pandemia. COVID-ig. Saúde mental.

ABSTRACT: The present study arises from the need to investigate the mental health of nursing professionals in the face of coping with COVID-I9, aiming to characterize professional exhaustion and identify the characteristics involved in nursing work on the front line of combating the pandemic that may influence in the occurrence of Burnout Syndrome. This research finds that symptoms of depression, anxiety and stress have been frequent among these workers, therefore, promoting the health of professionals is an extremely relevant issue, since these individuals need to be well to be able to provide a quality service the population. It is necessary to implement psychological intervention measures, such as: reorganizing the working day (allowing time for rest); appreciation and recognition for the service provided; improvement in remuneration; adequate provision of PPE and psychological assistance to promote mental well-being and a better quality of life for

\footnotetext{
I Graduanda de Enfermagem pela UniRedentor. E-mail: nataliaduartemıo@gmail.com

${ }^{2}$ Docente de Enfermagem da UniRedentor. E-mail: tiagopacheco20oo@yahoo.com.br
} 
health professionals exposed during the pandemic. It is concluded that it is extremely important to explore the possible ways to prevent Burnout, since the nurse is a professional of fundamental importance to fight the new coronavirus.

Keywords: Nursing. Burnout syndrome. Pandemic. COVID-19. Mental health.

\section{INTRODUÇÃO}

A pandemia provocada pelo Coronavírus (COVID-I9) foi reconhecida pela OMS no dia II de Março de 2020. De acordo com o Observatório da Enfermagem disponibilizado pelo COFEN (202I), do início da pandemia até o dia 27 de Outubro de 2021 foram contabilizados 869 óbitos entre a categoria dos profissionais de enfermagem, sendo 830 referentes a casos confirmados e 39 a casos suspeitos da doença.

Dentre os profissionais atuantes na linha de frente do cuidado ao paciente com COVID-ı9 a equipe de enfermagem é a mais vulnerável à doença, uma vez que a maior parte de seu trabalho envolve contato direto com pacientes e suas excretas. (DUPRAT \& MELO, 2020)

De acordo com Ribeiro, Vieira e Naka (2020, p.8) "O trabalho, a saúde e o adoecimento estão interligados à vida dos indivíduos, pois a atividade ocupacional reflete tanto na saúde mental quanto na física. Dessa maneira, ao mesmo tempo em que o trabalho é uma fonte de prazer, também pode se tornar um gerador de sofrimento."

No caso da enfermagem da linha de frente, a falta de equipamentos de proteção individual (EPIs) adequados, a sobrecarga de trabalho, o cansaço físico/mental e o estresse são alguns fatores que aumentam suas chances de adoecer. (DUPRAT \& MELO, 2020)

Devido as circunstâncias do atual cenário pandêmico os profissionais e serviços de saúde se depararam com a necessidade de se reinventar. Consultas e procedimentos eletivos precisaram ser postergados para dar prioridade às urgências e emergências, direcionando assim a atenção e os recursos disponíveis para o enfrentamento do vírus. (DUARTE; SILVA; BAGATINI, 202I) 
Segundo Gustmann de Castro et al (2021, p.2) a chegada do Coronavírus provocou uma dupla crise: primeiro no sistema de saúde mundial e depois nos protocolos necessários para o controle da infecção, que originaram uma crise econômica por interromper grande parte das atividades econômicas.

Houveram prejuízos para o ramo do turismo, da produção e produtividade, da cadeia de suprimentos, bolsas de valores e consequências econômicas entre países. Além disso, sobrecarregou serviços essenciais como farmácias, mercados e serviços de saúde, que tiveram que lidar com altas demandas de trabalho, além de vivenciarem o distanciamento social e o receio de contaminar seus familiares. (GUSTMANN DE CASTRO et al, 2021, p.2)

"Em momentos de maior pressão, a exemplo da luta contra o novo coronavírus, esses trabalhadores esquecem-se da própria saúde, propiciando o surgimento de transtornos relacionados ao estresse e à ansiedade como a Síndrome de Burnout (SB)." (BORGES et al, 202I)

Segundo Silva et al (2015) "a SB ainda é pouco conhecida entre a população geral. Sugestões de medidas preventivas e interventivas, voltadas aos profissionais da área de saúde, poderão refletir em mais qualidade na prestação dos serviços à população"

Sendo assim, questiona-se: Como a sobrecarga de trabalho ocasionada pela pandemia de Covid-ıg influenciou na ocorrência da Síndrome de Burnout nos profissionais da enfermagem? Logo, pretende-se identificar os fatores de risco para tal condição e as consequências que a mesma acarreta para o cuidado fornecido à população.

\section{METODOLOGIA}

O estudo caracteriza-se como uma revisão bibliográfica, contendo informações retiradas de fontes secundárias, com o intuito de reunir conhecimentos acerca da temática. As questões norteadoras para a produção científica foram: de que maneira a pandemia de COVID-rg influenciou na ocorrência da Síndrome de Burnout em profissionais de enfermagem? Quais as consequências do estresse e do desgaste para a assistência prestada? 
O levantamento bibliográfico necessário para responder a tais perguntas será obtido através das bases de dados Google Acadêmico e SciELO (Scientific Electronic Library Online), no período de Abril a Novembro de 202r. Descritores utilizados: Enfermagem; Pandemia; Covid-ı9; Síndrome de Burnout; Saúde mental. Os critérios de inclusão adotados foram: artigos disponíveis na íntegra, em português, publicados no período de 1999 a 2021 que respondam às questões pertinentes ao estudo. Os critérios de exclusão foram artigos que não envolvessem o objetivo da pesquisa.

\section{Pandemia de COVID-19}

Segundo a Organização Mundial da Saúde (OMS) uma pandemia se trata da disseminação mundial de uma nova doença. A partir do momento em que uma epidemia (surto que afeta uma região) se espalha por diferentes continentes com transmissão comunitária, ou seja, de pessoa para pessoa, o termo pandemia passa a ser utilizado. (SCHUELER, 202I)

"Em janeiro de 2020, pesquisadores chineses identificaram um novo vírus (SARS-CoV-2) como agente etiológico de uma síndrome respiratória aguda grave, denominada doença do coronavírus 2019, ou simplesmente COVID-ı̈" (CAVALCANTE et al, 2020)

"O primeiro caso da doença no Brasil foi confirmado em 26 de fevereiro de 2020. Tratava-se de um idoso residente em São Paulo/SP, que havia retornado de viagem à Itália. No dia 17 de março de 2020 ocorreu o primeiro óbito por Covid-ı9 no país.” (OLIVEIRA et al, 2020)

A pandemia provocada pelo Coronavírus foi reconhecida pela OMS no dia II de Março de 2020. (MALTA et al, 2020) Segundo dados fornecidos pela plataforma "Our World in Data" (202I), até final de Outubro de 202I foram relatadas mais de 5 milhões de mortes pela doença no mundo, sendo aproximadamente 608 mil apenas no Brasil.

Algumas das medidas implantadas no país afim de reduzir o número de casos de infecção e postergar o pico de contágio foram o distanciamento social, o cancelamento de aulas em todos os níveis de ensino e o isolamento social (quarentena). (NORONHA et al, 2020) 
Outras medidas fundamentais de prevenção orientadas pelo Ministério da Saúde incluem a lavagem das mãos com água e sabão ou higienização com álcool em gel, a prática de "etiqueta respiratória", que consiste em cobrir a boca e o nariz ao espirrar ou tossir, o não compartilhamento de objetos de uso pessoal, o hábito de manter os ambientes ventilados e o uso de máscaras, para que atuem como barreira à propagação do SARS-CoV-2. (OLIVEIRA et al, 2020)

A ampliação dos testes laboratoriais para rastrear e isolar os casos confirmados e a construção imediata de hospitais de campanha pela necessidade de expandir a quantidade de leitos disponíveis foram algumas das estratégias essenciais para o enfrentamento da pandemia. (NORONHA et al, 2020)

Aos 54 anos a enfermeira Mônica Calazans foi a primeira pessoa a ser vacinada contra a COVID-ı9 no Brasil. Ela recebeu o imunizante Coronavac no Hospital das Clínicas de São Paulo no dia 17 de Janeiro de 2021. (BADDINI \& FERNANDES, 202I)

Produzida pelo laboratório chinês Sinovac, a CoronaVac foi a primeira vacina contra o novo coronavírus aplicada no Brasil, testada pelo Instituto Butantan. Este imunizante segue um padrão diferente das outras vacinas, pois foi criado com base no próprio vírus inativado. (PINCER, 202I)

Antes da COVID-r9 a pandemia mais recente havia sido a da gripe suína em 2009, causada pelo vírus $H_{I} N_{I}$, que somou 36 mil casos em 75 países. Portanto, notase que a COVID-ı vem somar a uma enorme lista de disseminações que percorrem um longo período de tempo. (SCHUELER, 2021)

\section{SINDROME DE BURNOUT}

O Burnout é um transtorno psíquico de caráter depressivo associado a vida profissional do indivíduo que possui sintomas parecidos com os do estresse, da ansiedade e da síndrome do pânico. Se não tratado, pode evoluir para doenças físicas como: doença coronariana, problemas gastrointestinais, hipertensão, depressão e alcoolismo. (ALBUQUERQUE, 202I)

"Foi Identificado inicialmente nas áreas de cuidado/serviços e da educação e tradicionalmente definido como uma síndrome psicológica composta de três 
dimensões: exaustão emocional, despersonalização ou cinismo e baixa realização pessoal." (VIEIRA; RUSSO, 2019)

Koga et al (2015) descreve a exaustão emocional como um desgaste emocional e cansaço excessivo, resultando em falta de interesse e energia em relação ao trabalho. A despersonalização (ou cinismo) como o senso de distância emocional dos pacientes ou do trabalho e a baixa realização pessoal como correspondente ao sentimento de incompetência que está relacionado à baixa produtividade laboral, promovendo insatisfação profissional.

"Essa condição de esgotamento tem chamado a atenção dos profissionais da área médica do trabalho, que indicam a necessidade de maior atenção para os sintomas durante esse período de tensão e fadiga provocado pela pandemia de COVID-I9." (ALBUQUERQUE, 2021)

\footnotetext{
"O questionário Maslach Burnout Inventory (MBI) é o instrumento mais utilizado na investigação da doença, bem como na sua quantificação. É composto por 22 itens, distribuídos da seguinte maneira: nove itens relacionados à exaustão emocional, cinco à despersonalização e oito à baixa realização pessoal. Cada item marcado é classificado em uma escala de Likert de zero a seis, em que o = "nunca"; I = "algumas vezes por ano"; 2 = "uma vez por mês"; 3 = "algumas vezes por mês"; 4 = "uma vez por semana"; 5 = "algumas vezes por semana"; 6 = "todos os dias". O Burnout é, então, detectado segundo uma nota de corte para cada uma das três categorias: exaustão emocional 227 , despersonalização $\geq 10$ e baixa realização pessoal 233." (SILVEIRA et al, 2016)
}

A síndrome de Burnout no Brasil vem aumentando consideravelmente com o passar dos anos. Conforme dados do International Stress Management Association no Brasil (ISMA-BR), em 2019 um total de $72 \%$ da população economicamente ativa do país possuía elevados níveis de estresse. Destes, 32\% desenvolveram Burnout. A alta competitividade do mercado e a falta de tratamento precoce dos sintomas são algumas das razões para este aumento nos números de $\mathrm{SB}$ no país. (RIBEIRO; VIEIRA; NAKA, 2020)

"Em 2019 a Síndrome de Burnout foi incluída na Classificação Internacional de Doenças da Organização Mundial da Saúde, que entrará em vigor no ano de 2022.” (ALBUQUERQUE, 2021) 


\section{Relação da SB com a pandemia de COVID-ı9}

Os profissionais de saúde ao redor do mundo precisaram aprender a lidar com inúmeras dificuldades, tais como: o distanciamento de seus familiares para não arriscar contaminá-los; a perda de colegas de trabalho e conhecidos que vieram a óbito por conta da doença; as exaustivas jornadas de trabalho; o uso prolongado de EPIs que resultava, muitas vezes, em lesões; e principalmente a lidar com a morte sem critérios, que levava até mesmo jovens saudáveis. (ARAÚJO, 2020)

"A falta de autonomia, a sobrecarga de trabalho e a má definição do papel desses profissionais fazem com que a enfermagem seja a classe mais susceptível ao surgimento da SB." (RIBEIRO; VIEIRA; NAKA, 2020)

Segundo Maslach e Leiter (1999) trabalho de alta qualidade requer tempo e esforço, compromisso e criatividade, mas o indivíduo que se encontra em processo de desgaste já não está disposto a oferecer isso, logo, investe menos tempo e energia no trabalho, fazendo somente o que é totalmente necessário, o que acaba gerando maiores índices de falta e de acidentes de trabalho. (TRIGO; TENG; HALLAK, 2007 apud MASLACH; LEITER, 1999)

"Uma pesquisa inédita mostra que $83 \%$ dos profissionais de saúde
demonstram sinais da Síndrome de Burnout. Considerando o total da
pesquisa, incluindo os profissionais que estão e os que não estão na linha
de frente, a Síndrome do Burnout apareceu em $79 \%$ dos médicos; $74 \%$ dos
enfermeiros; e $64 \%$ dos técnicos de enfermagem. Os dados também
apontam que, quanto mais jovem o profissional, maior a chance de
esgotamento, e que a síndrome aparece mais em mulheres." (COREN -
MT, 2020)

Dentre os principais motivos para este aumento pode-se citar a imensa carga horária de trabalho, a baixa qualidade do sono, a diminuição drástica do convívio social, a falta de suporte emocional, os ganhos econômicos abaixo do esperado, a impossibilidade de fornecer o melhor aos pacientes (por conta de questões burocráticas e econômicas) a enorme responsabilidade e as cobranças em excesso. (MACHADO, 202I)

De acordo com o médico e presidente da Associação Brasileira de Empresas de Saúde e Segurança no Trabalho (ABRESST) Ricardo Pacheco é de suma importância que a saúde mental dos trabalhadores seja um dos itens de maior atenção 
por parte das empresas, que devem prover treinamento de líderes e criar novas ações afim de diminuir os problemas emocionais da equipe, acompanhando de perto a saúde de cada um dos funcionários. (ALBUQUERQUE, 202I)

Um estudo realizado na China com profissionais de saúde que cuidavam diretamente de pacientes com a infecção mostrou os seguintes resultados: de um total de 1.257 profissionais que trabalham em 34 hospitais (39\% médicos e $61 \%$ enfermeiros), aproximadamente 50\% apresentaram sintomas de depressão, 45\% de ansiedade, $34 \%$ de insônia e $72 \%$ de angústia. Apesar de tais dados não serem do Brasil, sustentam a hipótese de que durante uma crise como a atual, há uma maior probabilidade de que doenças relacionadas ao estresse, assim como a SB, venham a aumentar entre os profissionais da saúde. (RIBEIRO; VIEIRA; NAKA, 2020)

Segundo o Conselho Federal de Enfermagem (COFEN), o número de profissionais afastados no mês de abril de 2020 aumentou $660 \%$, saltando de 158 para I.203 enfermeiros e técnicos que se ausentaram dos seus postos de trabalho. (HUMEREZ; OHL; SILVA, 2020, p.5)

É necessário que os profissionais mantenham contato com familiares e amigos via rede social e evitem formas errôneas de se lidar com o estresse (como alcoolismo, tabagismo, má alimentação), priorizando rotinas mais saudáveis que envolvam ações de autocuidado, pausas para descanso entre os turnos de trabalho e pratica de atividade física regular nos tempos livres. (POLAKIEWICS, 2020)

"Este surto na saúde mental pode afetar diretamente as metas traçadas para a retomada econômica e para o fim da pandemia, sendo essencial o desenvolvimento de estratégias destinadas a fortalecer a saúde mental da população afetada." (RIBEIRO; VIEIRA; NAKA, 2020, p.7)

\section{CONSIDERAÇÕES FINAIS}

A qualidade do serviço prestado pelo profissional de enfermagem vai depender de seu bem-estar, portanto é necessário prezar pela saúde mental destes profissionais para que possam fornecer o melhor cuidado à população. Estudos referentes a prevalência da Síndrome de Burnout em profissionais da saúde ainda são escassos, principalmente em se tratando do momento atual de pandemia da COVID- 
19, a qual ainda vem sendo enfrentada, portanto, há uma baixa disponibilidade de produção acadêmica a respeito da temática. Entretanto, foi possível verificar que o distanciamento social, a convivência com a morte de colegas de trabalho, o medo de adoecer e de contaminar seus familiares, acompanhados dos sentimentos de solidão, insegurança, frustração e exaustão tiveram significativo impacto na saúde dos profissionais de enfermagem durante a pandemia, gerando insônia, ansiedade, depressão e caracterizando fatores contribuintes para o estabelecimento da Síndrome de Burnout. Desta forma, é de suma importância o acompanhamento da saúde mental destes profissionais por parte das instituições de saúde, uma vez que o enfermeiro é um profissional de fundamental importância para o combate ao novo coronavírus, além de se explorar as possíveis formas de se evitar o Burnout.

\section{REFERÊNCIAS BIBIOGRÁFICAS}

ALBUQUERQUE, Flávia. Excesso de trabalho e pandemia podem desencadear

Síndrome de Burnout. [online]. 202I. Disponível em: https://agenciabrasil.ebc.com.br/saude/noticia/2021-oI/excesso-de-trabalho-epandemia-podem-desencadear-sindrome-deburnout\#: :text=A\%20S\%C3\%ADndrome\%2ode\%2oBurnout\%20ou,doen\%C3\%A7as \%20mentais\%2orelacionadas\%20ao\%20trabalho Acesso em 20 de Outubro de 202I.

ARAÚJO, Juan Carlos Silva. Covid-ı: A doença que mostrou o protagonismo da enfermagem. [online]. 2020. Disponível em: https://pebmed.com.br/covid-I9-adoenca-que-mostrou-o-protagonismo-da-enfermagem/ Acesso em 3I de Outubro de 2021.

BADDINI, Bruna; FERNANDES, Daniel. Primeira pessoa é vacinada contra Covid-r9 no Brasil. CNN Brasil [online]. 2021. Disponível em: https://www.cnnbrasil.com.br/nacional/primeira-pessoa-e-vacinada-contra-covidI9-no-brasil/ Acesso em 3I de Outubro de 202I 
BORGES, Francisca Edinária de Sousa et al. Fatores de risco para a síndrome de Burnout em profissionais da saúde durante a pandemia de covid-19. Revista Enfermagem Atual In Derme [online] v. 95, n. 33, 2021. Disponível em: https://revistaenfermagematual.com/index.php/revista/article/view/835/79o Acesso em 04 de Junho de 202I

CAVALCANTE, João Roberto et al. COVID-ıg no Brasil: evolução da epidemia até a semana epidemiológica 20 de 2020. 2020. Epidemiologia e Serviços de Saúde [online]. v. 29, n. 4. Disponível em: https://doi.org/10.5123/Si679-49742020000400oio Acesso em 20 de Outubro de 2021.

COFEN, Conselho Federal de Enfermagem. Observatório da Enfermagem [online] 2021. Disponível em: http://observatoriodaenfermagem.cofen.gov.br/Acesso em II de Novembro de 2021.

COREN-MT, Conselho Regional de Enfermagem do Mato Grosso. Pesquisa aponta 83\% dos profissionais de saúde com Síndrome de Burnout: 'Desgastante' [online] 2020. Disponível em: http://mt.corens.portalcofen.gov.br/pesquisa-aponta-83-dosprofissionais-de-saude-com-sindrome-de-burnout-desgastante_I4939.html Acesso em 20 de Outubro de 2021.

DUARTE, Maria de Lourdes Custódio; SILVA, Daniela Giotti da; BAGATINI, Mariana Mattia Correa. Enfermagem e saúde mental: uma reflexão em meio à pandemia de coronavírus. Revista Gaúcha de Enfermagem [online]. 2021, v. 42. Disponível em: https://www.scielo.br/j/rgenf/a/MnRHwqvgq3kTrHQ2JPSLR7H/?lang=pt Acesso em 23 de Outubro de 2021.

DUPRAT, Irena Penha; MELO, Géssyca Cavalcante de. Análise de casos e óbitos pela COVID-19 em profissionais de enfermagem no Brasil. Revista Brasileira de 
Saúde Ocupacional [online]. 2020, v. 45. Disponível em: https://doi.org/10.1590/23176369000018220 Acesso em 31 de Outubro de 2021.

GUSTMANN DE CASTRO, Beatriz Leite et al. Empreendedorismo e coronavírus: impactos, estratégias e oportunidades frente à crise global. Estudios Gerenciales vol. 37, n. 158, p. 49-60, Mar. 2021 . Disponível em: http://www.scielo.org.co/scielo.php?script=sci_arttext\&pid=So123-59232021000100049 Acesso em i6 de Outubro de 2021.

HUMEREZ, Dorisdaia Carvalho de; OHL, Rosali Isabel Barduchi; SILVA, Manoel Carlos Neri da. Saúde mental dos profissionais de enfermagem do Brasil no contexto da pandemia Covid-ı: ação do Conselho Federal de Enfermagem. 2020. Cogitare enferm. [Internet]. Disponível em: https://docs.bvsalud.org/biblioref/2020/o7/ro99598/7-74115-v25-pt.pdf acesso em I8 de maio de 2021;

KOGA, Gustavo Kendy Camargo et al. Fatores associados a piores níveis na escala de Burnout em professores da educação básica. Cadernos Saúde Coletiva [online]. 2015, v. 23, n. 3. Disponível em: https://doi.org/10.159o/1414-462X201500030121 Acesso em 3I de Outubro de 202I.

MACHADO, Isadora Bazzan. Burnout: impacto da pandemia nos profissionais da saúde [online] 2021. Disponível em: https://www.sanarmed.com/burnout-impactoda-pandemia-nos-profissionais-da-saude-colunistas Acesso em 3I de Outubro de 202I.

MALTA, Deborah Carvalho et al. A pandemia da COVID-rg e as mudanças no estilo de vida dos brasileiros adultos: um estudo transversal, 2020. Epidemiol. Serv. Saúde, Brasília, v. 29, n. 4, e2020407, set. 2020. Disponível em: http://scielo.iec.gov.br/scielo.php?script=sci_arttext \&pid=Si67949742020000400025\&lng=pt\&nrm=iso Acesso em I6 de Outubro de 2021. 
NORONHA, Kenya Valeria Micaela de Souza et al. Pandemia por COVID-19 no Brasil: análise da demanda e da oferta de leitos hospitalares e equipamentos de ventilação assistida segundo diferentes cenários. Cadernos de Saúde Pública [online]. 2020, v. 36, n. 6. Disponível em: https://doi.org/ı.1590/oro2-311Xoori5320 Acesso em 3I de Outubro de 202I

OLIVEIRA, Wanderson Kleber de et al. Como o Brasil pode deter a COVID-ig. Epidemiologia e Serviços de Saúde [online]. 2020, v. 29, n. 2. Disponível em: https://doi.org/I0.5123/Si679-49742020000200023 Acesso em 20 de Outubro de 2021.

PINCER, Pedro. Entenda como é feita a Coronavac, primeira vacina contra covid-19 aplicada no Brasil. Senado Federal [online]. 2021. Disponível em: https://wwwi2.senado.leg.br/radio/r/noticia/202I/oI/20/entenda-como-e-feita-acoronavac-primeira-vacina-contra-covid-I9-aplicada-no-brasil Acesso em 31 de Outubro de 202I.

POLAKIEWICS, Rafael. Saúde mental de profissionais de enfermagem na pandemia de coronavírus. 2020. Disponível em: https://pebmed.com.br/saudemental-de-profissionais-de-enfermagem-na-pandemia-de-coronavirus/ Acesso em o6 de Junho de 202I.

RIBEIRO, Larissa Maciel; VIEIRA, Thayana de Almeida; NAKA, Karytta Souza. Síndrome de burnout em profissionais de saúde antes e durante a pandemia da COVID-19. Revista Eletrônica Acervo Saúde [online]. 2020. Disponível em: https://doi.org/ro.25248/reas.e502I.2020 Acesso em 3I de Outubro de 2021.

SCHUELER, Paulo. O que é uma pandemia, 202I. Disponível em: https://www.bio.fiocruz.br/index.php/br/noticias/i763-o-que-e-uma-pandemia Acesso em i6 de Outubro de 2021 
SILVA, Salvyana Carla Palmeira Sarmento et al. A síndrome de burnout em profissionais da Rede de Atenção Primária à Saúde de Aracaju, Brasil. Ciência \& Saúde Coletiva [online]. 2015, v. 20, n. Io. Disponível em: https://doi.org/I0.1590/1413-812320152010.19912014 Acesso em 21 de Outubro de 2021.

SILVEIRA, Ana Luiza Pereira da et al. Síndrome de Burnout: consequências e implicações de uma realidade cada vez mais prevalente na vida dos profissionais de saúde. Revista Brasileira de Medicina do Trabalho, 2016. Disponível em: http://www.rbmt.org.br/details/r2I/pt-BR/sindrome-de-burnout--consequencias-eimplicacoes-de-uma-realidade-cada-vez-mais-prevalente-na-vida-dos-profissionaisde-saude Acesso em or de Novembro de 2021.

TRIGO, Telma Ramos; TENG, Chei Tung; HALLAK, Jaime Eduardo Cecílio. Síndrome de burnout ou estafa profissional e os transtornos psiquiátricos. Archives of Clinical Psychiatry (São Paulo) [online]. 2007, v. 34, n. 5. Disponível em: https://doi.org/10.1590/Soror-60832007000500004 Acesso em 20 de Outubro de 2021. apud MASLACH, Christina; LEITER, Michael P. Trabalho: fonte de prazer ou desgaste: guia para vencer o estresse na empresa. Campinas; Papirus; 1999. 239 p.

VIEIRA, Isabela; RUSSO, Jane Araújo. Burnout e estresse: entre medicalização e psicologização. 2019. Disponível em: https://scielosp.org/article/physis/2019.v29n2/e290206/ Acesso em 02 de Junho de 2021. 